\title{
Fluid Overload in Critically III Children: More Questions Than Answers!
}

\author{
Nitin Dhochak ${ }^{1} \cdot$ Rakesh Lodha $^{1}$
}

Received: 16 December 2021 / Accepted: 21 December 2021 /Published online: 12 January 2022

(c) Dr. K C Chaudhuri Foundation 2022

Critically ill children are at risk of fluid retention, particularly in the first 24-48 $\mathrm{h}$ of admission due to need for extra fluid (for hemodynamic resuscitation, blood products, and infusions and medications), capillary leak and intravascular hypovolemia, and decreased water loss (minimal sweating, lack of insensible water loss in breathing in ventilated child, and decreased urine output due to acute kidney injury and syndrome of inappropriate antidiuretic hormone secretion). A meta-analysis of observational studies in children demonstrated association of fluid overload with mortality; for every $1 \%$ increase in fluid overload there was a $6 \%$ increase in mortality after adjusting for baseline disease severity [1]. Use of fluid boluses in impaired perfusion in resource-limited setting has been associated with increased mortality [2]. As such, there are no clinical trials in children on impact of conservative fluid management or deresuscitation strategies. But the recent recommendations on septic shock and ARDS suggest using conservative fluid approach to prevent fluid overload and complications.

In the study by Rameshkumar et al. in the Journal, impact of cumulative positive fluid balance during the first $7 \mathrm{~d}$ on various outcomes was assessed in a developing world PICU setting practising restrictive fluid protocol [3]. They did not observe an association of a positive fluid balance during the first $7 \mathrm{~d}$ with mortality, organ dysfunction, acute kidney injury or need for renal replacement therapy. The criteria for fluid overload used by the authors are at variance with earlier reports [1]. Also, the authors had a restrictive fluid and active deresuscitation approach, and maximal positive fluid balance in the cohort was only $3.1 \%$ [mean (SD): 1.52 (0.67) in group with positive fluid balance] [3].

Measurement of fluid balance is based on certain assumptions. Most commonly used approach uses difference of fluid input and output. Insensible losses are considered negligible

Rakesh Lodha

rakesh_lodha@hotmail.com

1 Department of Pediatrics, All India Institute of Medical Sciences, New Delhi 110029, India in this approach. Sweat-related losses are minimal with wellmaintained PICU temperature but respiratory water loss may be significant if the child is having respiratory distress and not being ventilated with heated humidified circuit. So, the fluid overload may be significantly overestimated with input-output method. Serial measurement of body weight is another approach; apart from the logistics, it does not take care of catabolism and weight loss during PICU stay, hence underestimates fluid overload. In a study of continuous renal replacement therapy initiation, median (IQR) fluid overload percentage calculated by input-output measurement was 14 $(6,32)$ compared to weight-based measurements $[8,(0,27)]$; though it was significantly higher among nonsurvivors in either method [4]. Errors in measurement tend to accumulate over time, so both these methods become unreliable for duration more than initial 3-5 d.

In the context of the current study [3], this implies that children classified as having 'positive fluid balance' may not practically have had any overload. As the children did not develop fluid overload, conclusions regarding effect of fluid overload on outcomes should not be made from this study. However, it is encouraging to note that fluid overload can be minimized using a protocol with conservative fluid approach. The authors had excluded the children with persistent hemodynamic instability at $48 \mathrm{~h}$, which is the group at highest risk of fluid overload and adverse outcomes. Also, it is not clear what proportion of PICU admissions were excluded due to above criteria and their outcome has not been described.

Experience from adult trials favor conservative fluid therapy [5]. Most modern PICU follow restrictive fluid approach. Trigger cutoffs (commonly used cutoff $>10 \%$ fluid overload) for starting deresuscitation measures are not definitive and are likely to change with the method used for fluid overload estimation. There is a need for clinical trials of effect of conservative fluid regimen to prevent fluid overload as well as deresuscitation measures (diuretics, albumin, or renal replacement therapy) on clinical outcome in critically ill children. 


\section{Declarations}

\section{Conflict of Interest None.}

\section{References}

1. Alobaidi R, Morgan C, Basu RK, et al. Association between fluid balance and outcomes in critically ill children: a systematic review and meta-analysis. JAMA Pediatr. 2018;172:257-68.

2. Maitland K, Kiguli S, Opoka RO, et al. Mortality after fluid bolus in African children with severe infection. N Engl J Med. 2011;364:2483-95.

3. Rameshkumar R, Chidambaram M, Bhanudeep S, et al. Prospective cohort study on cumulative fluid balance and outcome in critically ill children using a restrictive fluid protocol. Indian J Pediatr. 2021. https://doi.org/10.1007/s12098-021-03788-4.

4. Selewski DT, Cornell TT, Lombel RM, et al. Weight-based determination of fluid overload status and mortality in pediatric intensive care unit patients requiring continuous renal replacement therapy. Intensive Care Med. 2011;37:1166-73.

5. Silversides JA, Major E, Ferguson AJ, et al. Conservative fluid management or deresuscitation for patients with sepsis or acute respiratory distress syndrome following the resuscitation phase of critical illness: a systematic review and meta-analysis. Intensive Care Med. 2017;43:155-70.

Publisher's Note Springer Nature remains neutral with regard to jurisdictional claims in published maps and institutional affiliations. 